\title{
The Block Connectivity of Random Trees
}

\author{
Andrew R. A. McGrae Michele Zito \\ Department of Computer Science, \\ University of Liverpool \\ Ashton Street, Liverpool L69 3BX (UK). \\ e-mail: (andrew|michele)@liverpool.ac.uk \\ Submitted: Aug 27, 2008; Accepted: Dec 29, 2008; Published: Jan 7, 2009 \\ Mathematics Subject Classification: 05C 80
}

\begin{abstract}
Let $r, m$, and $n$ be positive integers such that $r m=n$. For each $i \in\{1, \ldots, m\}$ let $B_{i}=\{r(i-1)+1, \ldots, r i\}$. The $r$-block connectivity of a tree on $n$ labelled vertices is the vertex connectivity of the graph obtained by collapsing the vertices in $B_{i}$, for each $i$, to a single (pseudo-)vertex $v_{i}$. In this paper we prove that, for fixed values of $r$, with $r \geq 2$, the $r$-block connectivity of a random tree on $n$ vertices, for large values of $n$, is likely to be either $r-1$ or $r$, and furthermore that $r-1$ is the right answer for a constant fraction of all trees on $n$ vertices.
\end{abstract}

\section{Introduction}

A random tree on $n$ vertices, $\mathcal{T}_{n}$, is the typical element of the probability space defined over the $n^{n-2}$ labelled trees on $n$ vertices, assigning the uniform measure to each of its elements (see for instance [5] and references therein).

Let $r$ be a positive integer and define $m$ such that $r m=n$. Consider the partition $B_{1}, \ldots, B_{m}$ of $\{1, \ldots, n\}$ such that $B_{i}=\{r(i-1)+1, \ldots, r i\}$, for each $i \in\{1, \ldots, m\}$. If $T_{n}$ is a tree on $n$ vertices then its $r$-reduced graph $R_{r}\left(T_{n}\right)$ is a graph on $m$ vertices labelled $1,2, \ldots, m$ having an edge connecting vertices $i$ and $j$ for each edge in $T_{n}$ connecting a vertex $u \in B_{i}$ to a vertex $v \in B_{j}$. We will also say that $T_{n} r$-reduces to graph $G$ if $G=R_{r}\left(T_{n}\right)$. Note that $R_{r}\left(T_{n}\right)$, in general, may contain cycles. In what follows, $R_{r}\left(\mathcal{T}_{n}\right)$ will denote the $r$-reduced graph of a random tree on $n$ vertices. In a companion paper [4] we used random trees and their reduced graphs to study particular random instances of the empire colouring problem (a variant of the classical planar graph colouring problem defined by Heawood [2]). Here we are interested in the vertex connectivity of these graphs, for each fixed value of $r \geq 2$. Following [1, p. 9-10], a non-empty graph $G$ is connected if 
any two of its vertices are linked by a path and for any positive integer $d$, it is $d$-connected if $|V(G)|>d$ and $G-X$ is connected for any set $X \subseteq V(G)$ with $|X|<d$. Obviously, for each $r \geq 1, R_{r}\left(\mathcal{T}_{n}\right)$ is connected since the original tree is connected and any path in that graph is preserved in $R_{r}\left(\mathcal{I}_{n}\right)$. In fact, for $r \geq 2$, there may be multiple paths between any two vertices $u, v \in R\left(\mathcal{T}_{n}\right)$ since each consists of $r$ vertices in the underlying tree and each of the $r^{2}$ pairs of vertices $u_{i} \in B_{u}, v_{i} \in B_{v}$ are connected by a path in the tree. This suggests that $R_{r}\left(\mathcal{I}_{n}\right)$ will be $d$-connected for some $d$ depending on $r$. In the rest of this paper we make this intuition more precise. More specifically, first we argue that asymptotically almost surely (a.a.s.), that is with probability tending to one as $n$ tends to infinity, the connectivity of $R_{r}\left(\mathcal{T}_{n}\right)$ cannot be more than $r$. Then we show that, for each fixed $r \geq 2$, the probability that $R_{r}\left(\mathcal{T}_{n}\right)$ is not $r$-connected is lower bounded by a quantity that approaches a positive value, dependent on $r$ (but independent of $n$ ), as $n$ tends to infinity. Finally we argue that, a.a.s. the connectivity of $R_{r}\left(\mathcal{I}_{n}\right)$ is at least $r-1$. The first two results follow from a careful analysis of the properties of the vertices of degree $r$ in $R_{r}\left(\mathcal{T}_{n}\right)$. The last one, for $r \geq 3$, will be proved by estimating the number of trees whose $r$-reduced graph would be disconnected by the removal of a set $S$ of (at most) $r-2$ vertices and showing that this number is $o\left(n^{n-2}\right)$.

\section{Connectivity Upper Bounds}

Let $v$ be a vertex in $R_{r}\left(\mathcal{T}_{n}\right)$, we call $v$ a funny vertex if the degree of $v$ is $r$ and $v$ is incident to a pair of edges incident to the same two distinct vertices of $R_{r}\left(\mathcal{T}_{n}\right)$ (one of them being $v)$. From now on any such pair of edges will be called a double edge. In this section we study the number of vertices of degree $r$ in $R_{r}\left(\mathcal{T}_{n}\right)$, and among those, the number of funny vertices. Notice that the existence of a vertex $v$ of degree $r$ (resp. a funny vertex) immediately implies that the connectivity of $R_{r}\left(\mathcal{T}_{n}\right)$ cannot be larger than $r$ (resp. $r-1$ ) as in such case the removal of the neighbours of $v$ would leave $v$ as an isolated vertex.

\subsection{The Number of Vertices of Degree $r$ in $R_{r}\left(\mathcal{T}_{n}\right)$}

In this section we will use Chebyshev's inequality to prove that, for fixed values of $r \geq 1$, $N_{r, n}$ the number of vertices of degree $r$ in $R_{r}\left(\mathcal{T}_{n}\right)$ is well approximated by its expected value, in the sense that the probability that the (random) value of $N_{r, n}$ is far from $\mathbf{E} N_{r, n}$ tends to zero, as $n$ tends to infinity.

Lemma 1 Let positive integers $r$, and $n$ be given, with $r \leq n$. Then

$$
\begin{aligned}
& \mathbf{E} N_{r, n}=\frac{n}{r}\left(1-\frac{r}{n}\right)^{n-2} \\
& \mathbf{E} N_{r, n}^{2}=\mathbf{E} N_{r, n}+\left[\left(\frac{n}{r}\right)^{2}-\frac{n}{r}\right]\left(1-\frac{2 r}{n}\right)^{n-2} .
\end{aligned}
$$


Proof. Let $\mathcal{E}_{r}(v)$ denote the event " $\operatorname{deg}_{R_{r}\left(\mathcal{T}_{n}\right)}(v)=r$ ". We can write

$$
N_{r, n}=\sum_{v=1}^{m} I_{\mathcal{E}_{r}(v)}
$$

where $I_{\mathcal{E}_{r}(v)}$ is the random indicator for $\mathcal{E}_{r}(v)$. The number of labelled trees on $n$ vertices in which $r$ specific vertices are leaves is exactly $(n-r)^{n-2}$ (this is easily understood, say, via the correspondence between labelled trees and Prüfer codes [5]). Thus $\operatorname{Pr}\left[I_{\mathcal{E}_{r}(v)}=1\right]=$ $\left(1-\frac{r}{n}\right)^{n-2}$. The result on $\mathbf{E} N_{r, n}$ follows.

Also we may write

$$
\mathbf{E} N_{r, n}^{2}=\sum_{u, v=1}^{m} \operatorname{Pr}\left[I_{\mathcal{E}_{r}(v)}=1, I_{\mathcal{E}_{r}(u)}=1\right] .
$$

Given two distinct vertices $u, v \in V\left(R_{r}\left(\mathcal{T}_{n}\right)\right)$, the number of trees reducing to graphs in which both $u$ and $v$ have degree $r$ is equal to $(n-2 r)^{n-2}$. Hence

$$
\mathbf{E} N_{r, n}^{2}=\mathbf{E} N_{r, n}+\left[\left(\frac{n}{r}\right)^{2}-\frac{n}{r}\right]\left(1-\frac{2 r}{n}\right)^{n-2} .
$$

Theorem 1 Let $r$ be a fixed positive integer. Then

$$
N_{r, n}=\frac{n}{r}\left(1-\frac{r}{n}\right)^{n-2}+o(n) \quad \text { a.a.s. }
$$

Proof. For positive integers $r$ and $n$, with $n>r$,

$$
\left(1-\frac{2 r}{n}\right)^{n-2} \leq\left(1-\frac{r}{n}\right)^{2(n-2)}
$$

From this and Lemma 1, it is easy to see that

$$
\operatorname{Var} N_{r, n} \leq \mathbf{E} N_{r, n}
$$

The result now follows readily from Chebyshev's inequality.

\subsection{The Asymptotic Distribution of the Funny Vertices in $R_{r}\left(\mathcal{T}_{n}\right)$}

The result in Theorem 1 implies that the connectivity of $R_{r}\left(\mathcal{T}_{n}\right)$ is a.a.s. at most $r$. In this section we will further improve on this. Using the method of moments (see [3]) we find the asymptotic distribution of the number of funny vertices in $R_{r}\left(\mathcal{T}_{n}\right)$. This, in turns, implies that a simple upper bound on the probability that $R_{r}\left(\mathcal{T}_{n}\right)$ is $r$-connected approaches, as $n$ tends to infinity, a positive value smaller than one, dependent on $r$ (but independent of $n)$.

We start by stating a simple Lemma regarding the exponential function that will be used later. 
Lemma 2 For any real number $z$ such that $|z| \leq \frac{4}{7}$,

$$
\mathrm{e}^{z-z^{2}} \leq 1+z \leq \mathrm{e}^{z}
$$

Proof. The upper bound is obvious. For the other inequality, if $z>0$, note that, since $z<1$,

$$
\begin{aligned}
\mathrm{e}^{z} & \leq 1+z+\frac{z^{2}}{2}+z^{3}\left(\frac{1}{3 !}+\frac{1}{4 !}+\ldots\right) \\
& =1+z+\frac{z^{2}}{2}+z^{3}\left(\mathrm{e}-\left(1+\frac{1}{1 !}+\frac{1}{2 !}\right)\right)
\end{aligned}
$$

Hence,

$$
\begin{aligned}
\mathrm{e}^{z} & \leq 1+z+\frac{z^{2}}{2}+(\mathrm{e}-2.5) z^{3} \\
& \leq(1+z)+(1+z) \frac{z^{2}}{2} .
\end{aligned}
$$

If $z<0$ we can write $\mathrm{e}^{z}$ as $\mathrm{e}^{-|z|}$ and set $x=|z|$. We can thus write

$$
\mathrm{e}^{-x} \leq 1-x+\frac{x^{2}}{2}-\frac{x^{3}}{3 !}+\frac{x^{4}}{4 !}
$$

Now, since $1-\frac{x}{4} \geq \frac{3}{4}$ for $x<1$, we can write

$$
\frac{x^{3}}{3 !}-\frac{x^{4}}{4 !}=\frac{x^{3}}{3 !}\left(1-\frac{x}{4}\right) \geq \frac{x^{3}}{8}
$$

Therefore,

$$
\mathrm{e}^{-x} \leq 1-x+\frac{x^{2}}{2}-\frac{x^{3}}{8}=1-x+x^{2}\left(\frac{1}{2}-\frac{x}{8}\right) .
$$

Since $x \leq \frac{4}{7},\left(\frac{1}{2}-\frac{x}{8}\right) \leq(1-x)$ and so

$$
\mathrm{e}^{-x} \leq 1-x+x^{2}(1-x)
$$

In other words, since $z=-x$, we can write $\mathrm{e}^{-x}=\mathrm{e}^{z} \leq(1+z)\left(1+z^{2}\right)$. Along with the upper bound, this implies that $\mathrm{e}^{z} \leq(1+z) \mathrm{e}^{z^{2}}$. The lower bound follows by dividing both sides by $\mathrm{e}^{z^{2}}$

In what follows, $\operatorname{Po}(\lambda)$ will denote the Poisson probability law with average $\lambda$, and expressions like $S_{n} \stackrel{\mathrm{D}}{\rightarrow} \mathrm{Po}(\lambda)$, describe the fact that, as $n$ tends to infinity, the random variable $S_{n}$ converges in distribution to (a random variable with distribution) $\mathrm{Po}(\lambda)$. Let $F_{r, n}$ be the number of funny vertices in $R_{r}\left(\mathcal{T}_{n}\right)$. The following Lemma is key to our tight estimates of the $t^{t h}$ factorial moment of $F_{r, n}$, which in turn allow us to estimate the full distribution of $F_{r, n}$. 
Lemma 3 Let $r$ be a fixed positive integer with $r \geq 2$. For any set of $t>0$ vertices $v^{1}, \ldots, v^{t} \in V\left(R_{r}\left(\mathcal{T}_{n}\right)\right)$, the probability that $v^{1}, \ldots, v^{t}$ are funny vertices is

$$
\left(\begin{array}{l}
r \\
2
\end{array}\right)^{t} r^{t} \frac{(n-r t)^{n-2-t}}{n^{n-2}}(1+o(1))
$$

as $n$ tends to infinity.

Proof. For a vertex in $R_{r}\left(\mathcal{T}_{n}\right)$ to have minimum degree, each of its component tree vertices must be a leaf in $\mathcal{T}_{n}$, the number of trees in which $v^{1}, \ldots, v^{t}$ are funny is therefore equal to the number of trees on $n-r t$ vertices

$$
(n-r t)^{n-r t-2}
$$

multiplied by the number of ways to add $t$ groups of $r$ vertices as leaves such that in each group two vertices have parents in the same empire. For each group there are $\left(\begin{array}{l}r \\ 2\end{array}\right)$ choices for the two vertices that are to have parents in the same empire, and $r(n-r t)$ choices for the parent vertices. For each $1 \leq j \leq t$ the number of ways to choose the vertices within $v^{j}$ and their parents is therefore

$$
\left(\begin{array}{l}
r \\
2
\end{array}\right) r(n-r t)
$$

We now must count the number of ways to choose parents for the remaining $r-2$ vertices in each group, we can give an upper bound by allowing any remaining vertex in $v^{j}$ to choose any of the $(n-r t)$ vertices in the tree as its parent, giving a total of

$$
\left(\begin{array}{l}
r \\
2
\end{array}\right) r(n-r t)^{r-1}
$$

choices. This however, may overcount by counting trees more than once if there is more than one double edge incident to $v^{j}$. We therefore give a lower bound by counting only trees in which there is only one double edge and all other vertices have parents in different empires

$$
\left(\begin{array}{l}
r \\
2
\end{array}\right) r(n-r t) \prod_{l=1}^{r-2}(n-r t-l)=\left(\begin{array}{l}
r \\
2
\end{array}\right) r(n-r t)^{r-1}(1+o(1)) .
$$

It follows from (1) and (2) that the number of ways to add the rt vertices such that $v^{1}, \ldots, v^{t}$ are funny is

$$
\left(\begin{array}{l}
r \\
2
\end{array}\right)^{t} r^{t}(n-r t)^{r t-t}(1+o(1))
$$

the result follows by multiplying this by the number of trees on $n-r t$ vertices and dividing by $n^{n-2}$.

We are ready to state the main result of this section. 
Theorem 2 Let $r$ be a fixed positive integer, with $r \geq 2$. Then

$$
F_{r, n} \stackrel{\mathrm{D}}{\rightarrow} \mathrm{Po}\left(\left(\begin{array}{l}
r \\
2
\end{array}\right) \mathrm{e}^{-r}\right)
$$

as $n$ tends to infinity.

Proof. For integer $t \geq 1$, let $\mathbf{E}\left(F_{r, n}\right)_{t}$ be the $t^{t h}$ factorial moment of $F_{r, n}$. Then

$$
\mathbf{E}\left(F_{r, n}\right)_{t}=\sum_{v^{1}, \ldots, v^{t}}^{*} \operatorname{Pr}\left[v^{1}, \ldots, v^{t} \text { are funny vertices }\right],
$$

where the sum is over all $t$-tuples of distinct vertices $v^{1}, \ldots, v^{t} \in V\left(R_{r}\left(\mathcal{T}_{n}\right)\right)$. We can see that the number of ordered $t$-tuples is $\left(\frac{n}{r}\right)_{t}$, and by Lemma 3 the probability that all vertices are funny is

$$
\left(\begin{array}{l}
r \\
2
\end{array}\right)^{t} r^{t} \frac{(n-r t)^{n-2-t}}{n^{n-2}}(1+o(1)) .
$$

The $t^{t h}$ factorial moment of $F_{r, n}$ is therefore

$$
\mathbf{E}\left(F_{r, n}\right)_{t}=\left(\begin{array}{l}
r \\
2
\end{array}\right)^{t} \frac{(n-r t)^{n-2-t}}{n^{n-2-t}}(1+o(1)) .
$$

Using Lemma 2, we can bound (3) above by

$$
\left(\left(\begin{array}{l}
r \\
2
\end{array}\right) \mathrm{e}^{-r}\right)^{t}(1+o(1))
$$

and below by

$$
\left(\left(\begin{array}{l}
r \\
2
\end{array}\right) \mathrm{e}^{-r-\frac{r^{2}}{n}}\right)^{t}(1+o(1))
$$

The result follows, recalling that for a random variable $S_{n}$ depending on $n$, if $\lambda \geq 0$ is such that

$$
\mathbf{E}\left(S_{n}\right)_{t} \rightarrow \lambda^{t}
$$

for all $t \geq 1$ as $n$ tends to infinity, then $S_{n} \stackrel{\mathrm{D}}{\rightarrow} \operatorname{Po}(\lambda)$ (see [3, Corollary 6.8]).

Let $\phi_{r, n}(k)$ denote the probability that $R_{r}\left(\mathcal{T}_{n}\right)$ contains $k \geq 0$ funny vertices. If $R_{r}\left(\mathcal{T}_{n}\right)$ contains one or more funny vertices, then the removal of the $r-1$ neighbours of one of these vertices would disconnect the graph. The probability that $R_{r}\left(\mathcal{I}_{n}\right)$ is $r$-connected can therefore be bounded above by $\phi_{r, n}(0)$. The following result now is a direct consequence of Theorem 2.

Corollary 1 Let $r$ be a fixed positive integer with $r \geq 2$, and $n$ be a positive integer. Then the probability that the graph $R_{r}\left(\mathcal{T}_{n}\right)$ is r-connected is at most $\phi_{r, n}(0)$ and furthermore

$$
\phi_{r, n}(0) \rightarrow \exp \left\{-\left(\begin{array}{l}
r \\
2
\end{array}\right) \mathrm{e}^{-r}\right\}
$$

as $n$ tends to infinity. 

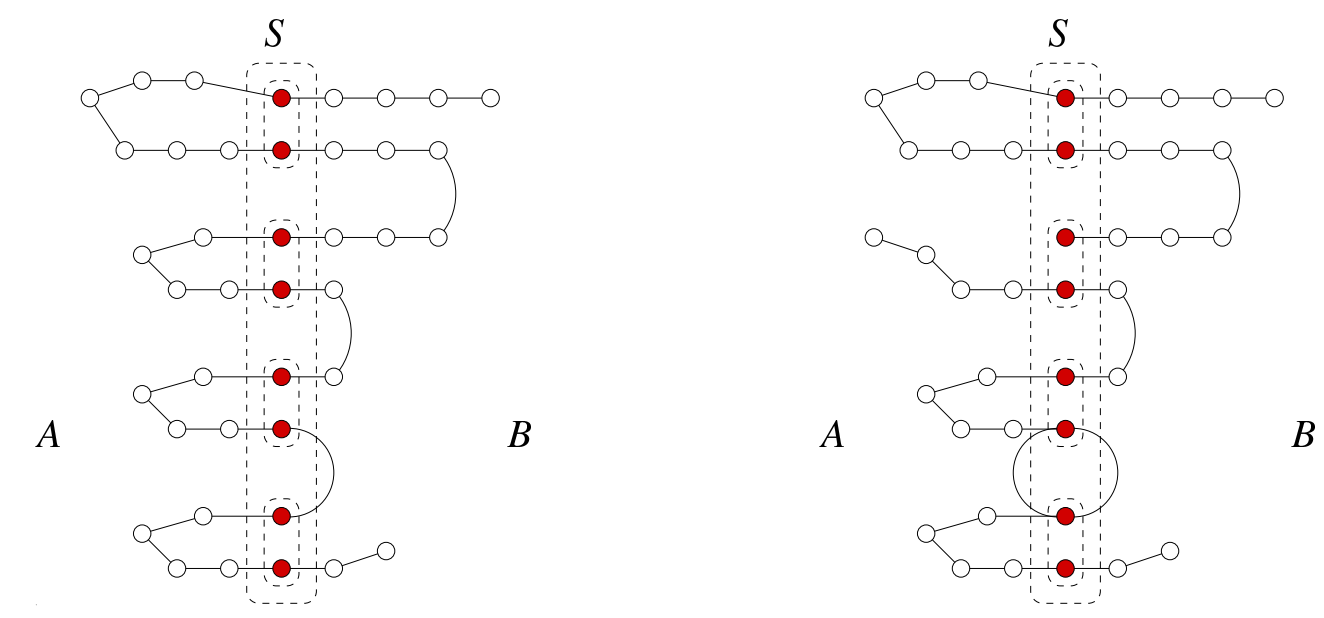

Figure 1: An example of $(A, B, S)$-arborescence (left) obtained from the $r$-reduced graph of a tree, with $r=2, d=4$, and $m=20$. The sets of blocks $S, A$, and $B$ are represented as sets of vertices. To avoid cluttering the picture only the four blocks of $S$ have been represented as rectangles enclosing two vertices each. The vertices of all blocks in $A$ are to the left of $S$, those blocks in $B$ are to the right of $S$. The example on the right-hand side describes a more general case in which $F_{A} \cup F_{B}$ is not a tree.

\section{Connectivity Lower Bound}

Let $m, r$ and $d$ be fixed positive integers and set $n=m r$. Let $G$ be a connected graph ${ }^{1}$ on $m$ vertices. If, for some $d<m-1, G$ is not $(d+1)$-connected, then there exists a partition of $V(G)$ into non-empty sets $A, B$, and $S$, such that ${ }^{2}|S|=d$ and all edges in the graph are either internal to one of the blocks or join a vertex in $S$ to a vertex in either $A$ or $B$. If $G$ is the $r$-reduced graph of some graph $H$ (the definition of $r$-reduced graph, given for trees in Section 1, readily generalizes to arbitrary graphs on $n$ vertices) and $G$ is not $d+1$-connected, the subgraph of $H$ induced by the vertices in the blocks in $A \cup S$ (resp. $B \cup S$ ) will be denoted by $F_{A}$ (resp. $F_{B}$ ) and will be such that each of its components contains at least one vertex in one of the blocks of $S$. Note that $F_{A} \cup F_{B}$ is not necessarily either connected or simple (see example on the right-hand side of Figure 1 ), however if $F_{A} \cup F_{B}$ is a tree (this is the case when $G=R_{r}\left(T_{n}\right)$ ), we call the pair $\left(F_{A}, F_{B}\right)$ an $(A, B, S)$-arborescence. We obtain an upper bound on the number of trees on $n$ vertices whose $r$-reduced graph would be disconnected by the removal of a set $S$ of vertices of size $d$ by estimating the total number of $(A, B, S)$-arborescences definable on a set of $n$ vertices.

Given positive integers $d, k$, and $n$, and positive integers $c_{1}, \ldots, c_{k}$ with $\sum_{i=1}^{k} c_{i}=d$,

\footnotetext{
${ }^{1}$ As we mentioned before, the $r$-reduced graph of a tree is always connected.

${ }^{2}$ We will only consider sets $S$ containing exactly $d$ vertices since if there is a smaller cut-set then any set formed from this by adding more vertices to it while leaving $A$ and $B$ non-empty will also disconnect the graph.
} 
let $h_{n, d}\left(c_{1}, \ldots, c_{k}\right)$ be the number of forests spanning a set $V$ of $n+d$ vertices with $k$ components such that, for each $i \in\{1, \ldots, k\}$, the $i^{\text {th }}$ component contains $c_{i}>0$ vertices in a given set $S \subseteq V$ of size $d$ and $x_{i}$ other vertices in $V \backslash S$. Then

$$
h_{n, d}\left(c_{1}, \ldots, c_{k}\right) \leq d ! \sum_{x_{1}, \ldots, x_{k}}\left(\left(\begin{array}{c}
n \\
x_{1}, \ldots, x_{k}
\end{array}\right) \prod_{i=1}^{k}\left(x_{i}+c_{i}\right)^{x_{i}+c_{i}-2}\right)
$$

where the sum is over all $k$-tuples of non-negative integers $x_{1}, \ldots, x_{k}$ summing to $n$.

The total number of $(A, B, S)$-arborescences on a set of $n$ vertices is at most

$$
Z_{n, r, d}=\sum_{b=1}^{\frac{1}{2}\left\lfloor\frac{n}{r}-d\right\rfloor}\left(\left(\begin{array}{c}
\frac{n}{r} \\
\frac{n}{r}-b-d, b, d
\end{array}\right) \sum_{\mathbf{c}^{A}, \mathbf{c}^{B}} h_{n-b r-d r, d r}\left(c_{1}^{A}, \ldots, c_{k_{A}}^{A}\right) h_{b r, d r}\left(c_{1}^{B}, \ldots, c_{k_{B}}^{B}\right)\right)
$$

where the inner sum is over all ways to choose two non-empty sequences of positive integers $c_{1}^{A}, \ldots, c_{k_{A}}^{A}$ and $c_{1}^{B}, \ldots, c_{k_{B}}^{B}$ adding up to $d r$. In the next section we will prove an upper bound on this quantity that is valid for fixed values of $r \geq 2$ and $d<r$, and sufficiently large values of $n$. This in turn leads to the following result, bounding the number of trees on $n=m r$ vertices whose $r$-reduced graph is $(r-1)$-connected. Its proof is deferred to the end of the forthcoming section.

Theorem 3 Let $r$ be a fixed positive integer with $r>1$. There exists a positive constant $C \leq((r-2) !)^{2} 2^{2 r(r-2)}(r-1)^{(r-1) r-2} r^{(r-1)^{2}-1}$ such that, for any fixed $\epsilon \in\left(0, \frac{r-1}{r}\right)$, if $n$ is sufficiently large, then the number of trees $T_{n}$ for which $R_{r}\left(T_{n}\right)$ is not $(r-1)$-connected is at most $C n^{n-3+\epsilon}$.

From Theorem 3, our result on the typical connectivity of $R_{r}\left(\mathcal{T}_{n}\right)$ follows as a simple corollary.

Corollary 2 For any fixed integer $r>1$, the $r$-reduced graph of a random tree on $n$ vertices is a.a.s. $(r-1)$-connected.

Proof. By the previous Theorem, the number of trees on $n$ vertices with $r$-reduced graphs that are not $(r-1)$-connected is at most $C n^{n-3+\epsilon}$ for some constant $C$. The probability that a random tree will have an $(r-1)$-connected $r$-reduced graph is therefore at least

$$
1-\frac{C}{n^{1-\epsilon}} \text {. }
$$

\subsection{Approximations and Proof Details}

To complete our proofs we need to work on $h_{n, d}\left(c_{1}, \ldots, c_{k}\right)$ first. 
Lemma 4 Let $k$ and $d$ be fixed positive integers. Then for any positive integer $n$, for all positive integers $c_{1}, \ldots, c_{k}$ with $\sum_{i=1}^{k} c_{i}=d$,

$$
\sum_{x_{1}, \ldots, x_{k}}\left(\begin{array}{c}
n \\
x_{1}, \ldots, x_{k}
\end{array}\right) \prod_{i=1}^{k}\left(x_{i}+c_{i}\right)^{x_{i}+c_{i}-2} \leq(n+d)^{n+d-2} .
$$

Proof. Consider the sets of vertices $W=\left\{w_{1}, \ldots, w_{n}\right\}, S=\left\{u_{1}, \ldots, u_{d}\right\}$ and for $0 \leq i \leq d$ let $d_{i}=\sum_{j=1}^{i} c_{j}$. Then,

$$
\left(\begin{array}{c}
n \\
x_{1}, \ldots, x_{k}
\end{array}\right) \prod_{i=1}^{k}\left(x_{i}+c_{i}\right)^{x_{i}+c_{i}-2}
$$

counts the number of trees $T_{1}, \ldots, T_{k}$, where for $1 \leq i \leq k$, the tree $T_{i}$ contains the vertices $u_{d_{i-1}+1}, \ldots, u_{d_{i}}$ and all vertices in $W_{i}$, given some arbitrary partition of $W$ into $k$ (possibly empty) subsets $W_{i}$. By summing over all $x_{1}, \ldots, x_{k}$ we consider all such partitions.

We can connect this sequence of trees by adding an edge $\left(u_{d_{i-1}+1}, u_{d_{i}+1}\right)$ for every $1 \leq i \leq k-1$ to obtain a tree $T$ with $n+d$ vertices. By construction, a different sequence of trees $T_{1}, \ldots, T_{k}$ leads to a different tree $T$. Thus we obtain that the number of different sequences of such trees $T_{1}, \ldots, T_{k}$ is less than or equal to the number of different trees $T$ with $n+d$ vertices, which is

$$
(n+d)^{n+d-2} \text {. }
$$

Let $r$ and $d$ be fixed positive integers, with $r>1$. For any positive integer $n$ define

$$
Y_{n, r, d}(a, b)=\left(\begin{array}{c}
\frac{n}{r} \\
a, b, d
\end{array}\right)(a r+d r)^{a r+d r-2}(b r+d r)^{b r+d r-2} .
$$

By (4) and Lemma 4,

$$
\left(\left(\begin{array}{c}
\frac{n}{r} \\
\frac{n}{r}-b-d, b, d
\end{array}\right) \sum_{\mathbf{c}^{A}, \mathbf{c}^{B}} h_{n-b r-d r, d r}\left(c_{1}^{A}, \ldots, c_{k_{A}}^{A}\right) h_{b r, d r}\left(c_{1}^{B}, \ldots, c_{k_{B}}^{B}\right)\right)
$$

is at most $(d !)^{2} Y_{n, r, d}(a, b)$. In what follows we will consider $Y_{n, r, d}(a, b)$ as defined on the set of positive integers $a$ and $b$ satisfying $a+b=\frac{n}{r}-d$.

Lemma 4 enables us to simplify our counting. $Z_{n, r, d}$ can be bounded above by $X_{n, r, d}\left(1, \frac{n}{2 r}-\frac{d}{2}\right)$ where

$$
X_{n, r, d}\left(b_{1}, b_{2}\right)=(d !)^{2} C \sum_{b=b_{1}}^{b_{2}} Y_{n, r, d}\left(\frac{n}{r}-b-d, b\right)
$$

and the positive constant $C$ is the number of ways to choose two non-empty sequences of positive integers $\mathbf{c}^{A}, \mathbf{c}^{B}$ each summing to $d r$, this is $2^{2 d r}$ by the binomial theorem. The remainder of our argument is a proof that this quantity is small compared with $n^{n-2}$. 
To prove Theorem 3 we will split $X_{n, r, r-2}\left(1, \frac{1}{2}\left\lfloor\frac{n}{r}-r+2\right\rfloor\right)$ into two parts:

$$
X_{n, r, r-2}\left(1, \frac{1}{2}\left\lfloor\frac{n}{r}-r+2\right\rfloor\right) \leq X_{n, r, r-2}\left(1,\left\lfloor n^{\epsilon}\right\rfloor\right)+X_{n, r, r-2}\left(\left\lfloor n^{\epsilon}\right\rfloor, \frac{1}{2}\left\lfloor\frac{n}{r}-r+2\right\rfloor\right)
$$

for some $\epsilon \in(0,1)$ to be chosen later. The following lemma shows that, for sufficiently large $n, Y_{n, r, d}(a, b)$ is maximised when either $a$ or $b$ is as large as possible. This fact will be used in turns to prove upper bounds on the two parts of $X_{n, r, r-2}\left(1, \frac{1}{2}\left\lfloor\frac{n}{r}-r+2\right\rfloor\right)$.

Lemma 5 Let $n$ be a positive integer, and $d$ and $r$ be fixed positive integers with $r>1$. Then,

$$
Y_{n, r, d}(a+1, b-1)>Y_{n, r, d}(a, b)
$$

for any integer $a$ and $b$ with $a>b \geq 1$, such that $a+b=\frac{n}{r}-d$.

Proof. For a fixed positive $d$,

$$
\begin{aligned}
\frac{Y_{n, r, d}(a+1, b-1)}{Y_{n, r, d}(a, b)} & =\frac{\left(\begin{array}{c}
\frac{n}{r} \\
a+1, b-1, d
\end{array}\right)}{\left(\begin{array}{c}
\frac{n}{r} \\
a, b, d
\end{array}\right)} \frac{(a r+d r+r)^{a r+d r+r-2}}{(a r+d r)^{a r+d r-2}} \frac{(b r+d r-r)^{b r+d r-r-2}}{(b r+d r)^{b r+d r-2}} \\
& =\frac{1}{a+1} \frac{(a+d+1)^{(a+1) r+d r-2}}{(a+d)^{a r+d r-2}} b \frac{(b+d-1)^{(b-1) r+d r-2}}{(b+d)^{b r+d r-2}} .
\end{aligned}
$$

Define the function $f(x)$, for $x \geq 0$, as

$$
f(x)=\frac{1}{x+1} \frac{(x+d+1)^{(x+1) r+d r-2}}{(x+d)^{x r+d r-2}},
$$

then (6) is equal to

$$
f(a) f(b-1)^{-1}
$$

The statement of this Lemma therefore holds if $f(x)$ is strictly monotone increasing for $x>0$. The first derivative of $f(x)$ has the same sign as

$$
r \log \left(1+\frac{1}{x+d}\right)+\frac{1-d-d x-x^{2}}{(x+d+1)(x+d)(x+1)} .
$$

Using Lemma 2 we can bound this below by

$$
\frac{(r-1) x^{3}+(r+2(r-1) d) x^{2}+(2 d-1)(r-1) x+\left(d^{2}-1\right) r+d}{(x+d+1)(x+d)(x+1)} .
$$

For positive $x, d$ and $r$, every bracketed term in the last expression is non-negative and so $f^{\prime}(x)>0$ for all $x>0$. Hence $f(x)$ is strictly monotone increasing for $x>0$ and the result follows. 
Proof of Theorem 3. For $r=2$ the result is obvious since trees are connected graphs. For $r>2$, we give an upper bound on $Z_{n, r, d}$ and hence on the number of trees $T_{n}$ for which the vertex set of $R_{r}\left(T_{n}\right)$ can be split in three sets $A, B$ and $S$ with $|S|=r-2$, $|B|=b$ for some $b \in\left\{1, \ldots, \frac{1}{2}\left\lfloor\frac{n}{r}-r+2\right\rfloor\right\}$, and $|A|=\frac{n}{r}-b-(r-2)$, and such that there are no edges connecting $A$ to $B$. First note that

$$
X_{n, r, r-2}\left(1, \frac{1}{2}\left\lfloor\frac{n}{r}-r+2\right\rfloor\right) \leq X_{n, r, r-2}\left(1,\left\lfloor n^{\epsilon}\right\rfloor\right)+X_{n, r, r-2}\left(\left\lfloor n^{\epsilon}\right\rfloor, \frac{1}{2}\left\lfloor\frac{n}{r}-r+2\right\rfloor\right) .
$$

Lemma 5 allows us to bound $X_{n, r, r-2}\left(1,\left\lfloor n^{\epsilon}\right\rfloor\right)$ above by making $A$ as large as possible in each term

$$
X_{n, r, r-2}\left(1,\left\lfloor n^{\epsilon}\right\rfloor\right) \leq C n^{\epsilon}\left(\left(\begin{array}{c}
\frac{n}{r} \\
\frac{n}{r}-r+1,1, r-2
\end{array}\right)(n-r)^{n-r-2}((r-1) r)^{(r-1) r-2}\right) .
$$

The multinomial coefficient $\left(\begin{array}{c}\frac{n}{r} \\ \frac{n}{r}-r+1,1, r-2\end{array}\right)$ is at most $\left(\frac{n}{r}\right)^{r-1}$, thus

$$
\begin{aligned}
X_{n, r, r-2}\left(1,\left\lfloor n^{\epsilon}\right\rfloor\right) & \leq((r-2) !)^{2} 2^{2 r(r-2)} n^{\epsilon}\left(\left(\frac{n}{r}\right)^{r-1}(n-r)^{n-r-2}((r-1) r)^{(r-1) r-2}\right) \\
& \leq C^{\prime} n^{n-3+\epsilon}
\end{aligned}
$$

for some constant $0<C^{\prime} \leq((r-2) !)^{2} 2^{2 r(r-2)}(r-1)^{(r-1) r-2} r^{(r-1)^{2}-1}$.

Next we look at $X_{n, r, r-2}\left(\left\lfloor n^{\epsilon}\right\rfloor, \frac{1}{2}\left\lfloor\frac{n}{r}-r+2\right\rfloor\right)$. This part of $X_{n, r, r-2}\left(1, \frac{1}{2}\left\lfloor\frac{n}{r}-r+2\right\rfloor\right)$ still contains a large number of terms, but each term is relatively small. By Lemma 5 moving vertices from $B$ to $A$ will increase the size of $Y_{n, r}\left(\frac{n}{r}-b-(r-2), b, r-2\right)$. We can therefore bound $X_{n, r, r-2}\left(\left\lfloor n^{\epsilon}\right\rfloor, \frac{1}{2}\left\lfloor\frac{n}{r}-r+2\right\rfloor\right)$ above by

$$
\begin{aligned}
& ((r-2) !)^{2} 2^{2 r(r-2)} \frac{n}{2 r} \times \\
& \quad\left(\left(\begin{array}{l}
\frac{n}{r} \\
\frac{n}{r}-r-\left\lfloor n^{\epsilon}\right\rfloor+2,\left\lfloor n^{\epsilon}\right\rfloor, r-2
\end{array}\right)\left(n-r\left\lfloor n^{\epsilon}\right\rfloor\right)^{n-r\left\lfloor n^{\epsilon}\right\rfloor-2}\left(r\left\lfloor n^{\epsilon}\right\rfloor+r^{2}-r\right)^{r\left\lfloor n^{\epsilon}\right\rfloor+r^{2}-r-2}\right) .
\end{aligned}
$$

In the expression above, the multinomial coefficient is at most $\left(\frac{n}{r}\right)^{\left\lfloor n^{\epsilon}\right\rfloor+r-2}$, and thus we get (for $n$ sufficiently large)

$$
X_{n, r, r-2}\left(\left\lfloor n^{\epsilon}\right\rfloor, \frac{1}{2}\left\lfloor\frac{n}{r}-r+2\right\rfloor\right) \leq((r-2) !)^{2} 2^{2 r(r-2)} r^{r^{2}} r^{(r-1) n^{\epsilon}} n^{n-2+\epsilon\left(r^{2}-r-2\right)-(r-1-\epsilon r) n^{\epsilon}} .
$$

For $r \geq 2$ and $\epsilon<\frac{r-1}{r}$, this means that

$$
X_{n, r, r-2}\left(\left\lfloor n^{\epsilon}\right\rfloor, \frac{1}{2}\left\lfloor\frac{n}{r}-r+2\right\rfloor\right) \leq C^{\prime \prime} n^{n-3} .
$$

for some constant $0<C^{\prime \prime} \leq((r-2) !)^{2} 2^{2 r(r-2)} r^{r^{2}}$. The result follows by adding together (7) and (8). 
Acknowledgements. The authors are endebted to the anonymous referees for pointing out a mistake in our original counting of the number of $(A, B, S)$-arborescences, and, in general, for the thorough reading of the manuscript that lead to a number of comments that have helped to significantly improve the quality of the presentation.

\section{References}

[1] R. Diestel. Graph Theory 2nd edition, Springer-Verlag, New York, 2000.

[2] P. J. Heawood. Map colour theorem, Quarterly Journal of Pure and Applied Mathematics, 24:332-338, 1890.

[3] S. Janson, T. Luczak, A. Ruciński. Random Graphs, John Wiley \& Sons, 2000.

[4] A. R. A. McGrae, M. Zito. Colouring Random Empire Trees, In E. Ochmański and J. Tyszkiewicz, editors, Mathematical Foundations of Computer Science 2008, volume 5162 of Lecture Notes in Computer Science, pages 515-526. Springer-Verlag, 2008.

[5] J. W. Moon. Counting Labelled Trees, volume 1 of Canadian Mathematical Monographs, Canadian Mathematical Congress, 1970. 